\title{
Lecciones desde la planificación territorial y reconstrucción post desastre en Armenia, Colombia
}

Jabir Rodríguez-Rodríguez. Universidad Autónoma de Manizales, Manizales, Colombia.

Estas notas revisan el impacto del sismo que sacudió la ciudad de Armenia, Colombia, en 1999, un 25 de enero; además, desarrolla la respuesta institucional del Estado colombiano, tratándose de uno de los mayores desafíos que se le ha planteado a sus relaciones con la sociedad civil en región alguna del país. A renglón seguido, se examinan los procesos de planificación y resignificación del espacio y la manera como la reconstrucción se convirtió en una oportunidad para el desarrollo sostenible, desde una perspectiva urbanística contemporánea. Finalmente, se discute sobre la dimensión política y la participación ciudadana, elementos que tienen que servir para la integración social y para desarrollar proyectos sustentables y adaptados a las características concretas de cada territorio. Nos referimos a una participación que genere inclusión social, integrada a una creciente expansión de la ciudadanía, donde actuemos como seres sociales y no individuales, en el marco de la dimensión política de la solidaridad.

\section{El contexto de la tragedia urbana}

El sismo, de gran intensidad, ${ }^{1}$ ocurrido el 25 de enero de 1999 a la 1:19 P.M. en la región del Eje Cafetero, afectó de manera grave la vida, la economía, la estructura urbana de las ciudades y las dinámicas políticas, sociales y culturales de la población. Siendo considerado el mayor desastre urbano en la historia nacional, dejó al descubierto la fragilidad de la economía cafetera, la crisis político-administrativa y financiera de municipios y departamentos, la obsolescencia

1 El 25 de enero de 1999, dos eventos sísmicos significativos afectaron un área estimada en 1.360 kilómetros cuadrados. El primero, denominado por los expertos del Instituto de Investigaciones en Geociencias, Minería y Química (Ingeominas) como el evento principal, se desarrolló a las 13:19 horas (18:19 GMT), localizándose en los 4,41 de latitud norte y los $75,72^{\circ}$ de longitud oeste. La misma fuente estimó la profundidad del sismo en alrededor de $10 \mathrm{~km}$, con una magnitud en la escala de Richter de 6,2. Con posterioridad al evento principal, a las 17:40 horas (22:40 GMT) se registró una réplica significativa de 5,8 en la escala Richter, algo desplazada al sur: latitud norte $4,39^{\circ}$. Un número considerable de réplicas afectó el área en las semanas siguientes: 138 durante el primer mes, con un campo que se ha extendido a una superficie de $300 \mathrm{~km}^{2}$, y magnitudes que en algunos casos alcanzan los 4,4 en la escala de Richter.

E-mail: rodriguezrodriguezjahir@gmail.com 
de liderazgos y la corrupción del aparato institucional; en fin, las múltiples vulnerabilidades que le asistían a la región.

La magnitud de la tragedia no tenía antecedentes en el país. Se presentó en 28 municipios, 450.000 personas fueron afectadas de manera directa, 14.000 viviendas fueron destruidas totalmente y más de 80.000 sufrieron daños severos. La mayor parte de la infraestructura de servicios públicos, como acueductos, redes telefónicas, centros escolares, sedes hospitalarias y administrativas, sufrieron un colapso total, así como miles de instalaciones productivas como beneficiade$\operatorname{ros}^{2}$ de café y talleres, también almacenes y locales comerciales.

La reconstrucción de la región del desastre se presentó, entonces, como un gran reto al Estado colombiano y uno de los mayores desafíos que se les ha planteado a sus relaciones con la sociedad civil en región alguna del país. Esta reconstrucción debía obedecer, además, a un país en crisis con pérdida de legitimidad institucional, depresión económica y fiscal, con niveles críticos de confrontación armada; por lo tanto, debía responder a la necesidad de convocar y vincular múltiples intereses en función de un propósito común.

Frente a la debilidad institucional imperante en diferentes niveles del Estado y la inoperancia que en su momento mostraron los organismos de prevención y atención de desastres, el gobierno nacional buscó corresponder a la situación con el diseño de una estrategia de intervención y la creación de un instrumento de gestión que fuera ágil, eficiente y transparente, y que se pusiera al frente del proceso de la reconstrucción. Por ello se creó el Fondo para la Reconstrucción y Desarrollo Social del Eje Cafetero (Forec).

Lo anterior se tradujo en lo que se ha dado en llamar el modelo de intervención para la reconstrucción del Eje Cafetero. El o los modelos constituyen una simplificación de un sistema determinado o la simulación del establecimiento de una estructura lógica, que busca en diferentes campos enfrentar una situación dada; en este caso, el sistema está referido a la gestión pública, redefinida o reestructurada en función de llevar a cabo una gran intervención de naturaleza inusitada, con participación de la sociedad civil.

En las calles de las ciudades y pueblos quedó al desnudo nuestra sociedad, la impresionante corrupción no solo del Estado, sino de la mayoría de sus estamentos; en una palabra, se pudo descubrir cómo se ha supeditado lo público a los mezquinos intereses egoístas, vestidos - ellos sí- de poderosos privilegios.

Pero el terremoto despertó igualmente en la población afectada sus más bellos sentimientos. De las ruinas de sus casas salió la mano amiga, se escuchó la voz del vecino, se compartió el dolor propio y el ajeno y se volvió a sentir de una manera colectiva, no solo de palabra sino de acto, la energía común que permite la acción solidaria. Salió la fuerza comunitaria, la posibilidad de construir un espacio con nuevos vínculos de convivencia en el trabajo de reconstruir lo propio y lo ajeno, en el trabajo y la vida en común; surgieron inéditas formas de participación en los procesos que definen lo fundamental de la vida colectiva, la capacidad de crear lo propio, de crear aquello en lo que un colectivo puede reconocerse: su morada, su espacio vital, el que permite la capacidad de ser, 
sentir y pensar en los problemas de nuestra existencia no solo individual, sino colectiva.

De los escombros salió la miseria de los privilegios y la riqueza de la participación comunitaria, la participación ciudadana, la de la gente corriente, en todas aquellas cosas que afectan su vida económica, política y social; salió la posibilidad real de discutir, de organizarse y soñar, de ampliar el espacio de lo público deliberando sobre las cosas más sencillas y las más complejas.

El terremoto hizo colapsar buena parte de la institución social de la región afectada, pero en especial de la ciudad de Armenia. Nos mostró cómo el país no puede seguir aplazando las grandes decisiones públicas; cómo debe reconstruir el Estado y liberar de temores y amarraduras a la sociedad civil, permitiendo que fluya la posibilidad de que en nuestro medio exista un sistema político donde podamos concertar con la mayoría de la población, nuestras decisiones colectivas.

El terremoto mostró cómo la sociedad se puede organizar por sí misma casi sin la ayuda del gobierno, el cual no tiene una clara respuesta política. Reveló cómo las soluciones para los grandes problemas son políticas y en ellas el papel de la sociedad es trascendente. Como dijo un anónimo habitante de la ciudad, convertido por la desgracia en ciudadano: "Oíste, vos, si nos organizamos, la iniciativa es lo que diga la comunidad, no el gobierno".

Si cuando los muros destrozados por el sismo dejaron desnudos en la calle a los habitantes de la ciudad, estos, con su más preciado don, el habla, pudieron comunicar su sufrimiento común, haciendo emerger la más inaudita de las fuerzas: la fuerza de lo colectivo. Hicieron recordar que lo más importante de la existencia no es la propiedad de las cosas, la posesión de pequeños bienes, sino el ser, el ser humano que desata el poder de la comunicación indicándonos cómo en materia lingüística nadie es propietario; cómo con el habla nos acercamos, y cómo nos hacemos menos frágiles a través de la comunicación y la solidaridad que ella permite.

La situación que se vivió en la ciudad, donde en un espacio y en un tiempo reducidos se dio un germen de autonomía, nos ofreció una lección esencial, en cuanto abrió una ventana de esperanza para construir una nueva vida política no solo en el Eje Cafetero, sino en el país: se requirió de una democracia más próxima, con una intervención activa, organizada y legal, liderada por la comunidad en la discusión sobre los asuntos que son de su conveniencia. Este pequeño espacio de autonomía no surgió de una forma fácil. Se lo ha querido adjudicar al modelo desarrollado a través del Forec, que sin duda ayudó al dejar hacer, pero el nuevo espacio fue el que se gestó con la participación comunitaria. Allí el pueblo, por su propia iniciativa, vigiló y exigió el cumplimiento de lo pactado con las instituciones de la ciudad. Y con ello nos reveló cómo las crisis se profundizan innecesariamente a causa de la inacción y la falta de capacidad y confianza de las instituciones para llevar a cabo los cambios de política requeridos para construir nuevamente lo público.

La reconstrucción física de la ciudad se convirtió en un lugar de participación comunitaria, abriendo la posibilidad de formar ciudadanía a fuerza de la desgracia; hizo descubrir la potencia de la convivencia común por medio de la organización ciudadana, y de la creatividad y apoyo desplegados por la unidad de participación comunitaria del Forec y de algunas ONG. El terremoto nos reveló cómo el ser humano logra sobre- 
vivir creando solidaridad a través de la existencia en común; cómo la fuerza de la convivencia permite subsistir a un penoso drama. Y cómo el vivir como ciudadanos, partícipes de nuestros actos, búsquedas, errores y aciertos, hace posible crear nuevamente un sentido para la vida.

Esto fue lo que ocurrió en Armenia después del terremoto. Se vivió un espacio de autonomía, un momento esperanzador salido de los escombros del derrumbe físico. De donde aparentemente no surgía sino dolor y pánico, emergió la solidaridad común. Y ese drama de desolación y muerte se transformó en creación social: las gentes que vivieron esta tragedia son un modelo, un modelo para reconstruir al país mostrándonos cómo, a través de los lazos de apoyo y la participación, podemos levantar la infraestructura social de nuestro futuro: la creación de una nueva sociedad, más justa, equitativa y solidaria, viviendo en paz, tratando de conciliar la justicia con la libertad.

\section{Impacto del sismo}

El Eje Cafetero parecía tener buenos niveles de equidad, pero la realidad de la miseria en que vivían muchos de sus habitantes quedó al descubierto al caerse las casas de inquilinato y descubrirse las muy precarias condiciones habitacionales en que vivían miles de familias que no eran propietarias de esos inmuebles.

Con el sismo se afectaron 28 municipios de los departamentos de Quindío, Risaralda, Tolima, Valle y Caldas. Más de mil personas murieron, cerca de 8.000 resultaron heridas y 100.000 inmuebles resultaron afectados, de los cuales 31.000 no son reparables. En la zona rural se dañaron total o parcialmente cerca de 10.000 viviendas y 5.000 beneficiaderos. También se produjeron múltiples daños en la infraestructura social de servicios públicos domiciliarios y en el sistema ambiental de la zona. Con el sismo sobrevinieron intensos desplazamientos intramunicipales y nuevas ocupaciones del territorio.

Atendiendo a los estimativos del Forec, los efectos en materia de vivienda y otras edificaciones, tanto urbanas como rurales, ascendieron a $\$ 1,6$ billones (pesos colombianos). El impacto en la infraestructura de educación y cultura se calculó en $\$ 206.000$ millones y en salud, en $\$ 90.000$ millones. Los daños en vías y transporte se estimaron en $\$ 77.000$ millones, mientras que la afectación en los sistemas de acueductos y alcantarillados sumó \$29.000 millones, y en aseo y energía, $\$ 43.000$ millones. La medición de los costos económicos de los daños ambientales presentó dificultades. Las pérdidas en la producción correspondieron al 0,19\% del PIB, una cifra pequeña en el conjunto nacional, pero de gran significación en el contexto regional.

La Comisión Económica para América Latina y el Caribe (Cepal) calculó que los daños directos del terremoto fueron del orden de $\$ 2,1$ billones (correspondientes al 1,4\% del PIB nacional). Para el gobierno nacional, el costo de la intervención a través del Forec tuvo un valor aproximado de $\$ 1,7$ billones.

Para algunos observadores de estos temas, como producto del sismo también se consideró probable que la fuerte tendencia a incrementar su población que traían los municipios de Armenia ${ }^{3}$ y Calarcá (que coincidentemente fueron los de mayor afectación) se suavizara

3 La tasa de crecimiento de Armenia entre 1983 y 1995 fue de 3,5\%, levemente inferior a la de Pereira, que creció al 3,6\%. Pero se estimaba que en 1997 ya aquella ciudad tendría una tasa (2,5\%) superior a la de esta última (2,3\%). 
un poco, y que a cambio se incrementara la presión demográfica hacia Pereira-Dosquebradas. De hecho, ya son visibles las tendencias migratorias y, por ejemplo, la Alcaldía de Armenia estimaba que por efecto del terremoto llegaron a esta ciudad entre febrero y diciembre de 1999 no menos de 40.000 personas.

Así pues, si bien este conjunto urbano regional había tenido en el momento del sismo una ligera pérdida de dinámica poblacional, desarrollaba complejos procesos de relación urbana cuyo papel funcional había sido visualizado como centro de producción, comercialización y de servicios para la zona cafetera.

De esta manera, en el comportamiento poblacional seguirán influyendo, entre otros, factores como el modelo de desarrollo económico, la pérdida de importancia relativa de las actividades cafeteras e industriales, el progresivo empobrecimiento de la población, el fortalecimiento de una red vial principal, el escalonamiento de la violencia en el país y la subsecuente llegada a la región de familias desplazadas y, por supuesto, la ejecución real de los planes zonales de la reconstrucción.

\section{La respuesta institucional: un nuevo modelo de gestión para la reconstrucción}

Una vez ocurrido el sismo, el Presidente de la República decretó la emergencia, creó el Forec, conformó y nombró su Consejo Directivo a través de los Decretos 197, 198 y 199 de enero 31 de 1999. El Fondo fue instaurado para la reconstrucción del Eje Cafetero, como una entidad de naturaleza especial de orden nacional con sede en Armenia, dotada de personería jurídica, autonomía patrimonial y financiera, sin estructura administrativa propia y cuyo objetivo consistió en la financiación y realización de las actividades necesarias para la reconstrucción económica, social y ecológica de la región. Para hacer operativo el proceso, se nombró un Consejo Directivo conformado por representantes del sector privado, de los gobiernos territoriales y del Departamento Nacional de Planeación (DNP), que hizo la Secretaría Técnica. Durante la vigencia del Fondo, dicho Consejo se reunió en promedio 2,5 veces al mes, de lo cual quedó constancia a través de actas.

Desde la Presidencia de la República se especificaron los elementos centrales para implementar y poner en funcionamiento un nuevo modelo de gestión que se encargara de la reconstrucción, para lo cual estableció los principales lineamientos, los cuales se describen a continuación.

\section{Principios de política para el modelo de la reconstrucción}

El proceso de reconstrucción propuso devolver a las gentes de la región afectada unas condiciones de vida digna, a través de la aplicación de los recursos a proyectos que promovieran su desarrollo. Lo hizo a partir de ocho principios básicos, los cuales se interpretan con base en el manejo que el monitoreo tiene de la información y los análisis propios de la experiencia (Gómez, 2001a).

- Integralidad del desarrollo. Se refiere a la necesidad de planificar y emprender acciones en forma "suprasectorial"; por lo tanto, reclama una visión sistémica de cada una de las localidades y áreas físicas por intervenir; incluye la gestión en los ámbitos de la infraestructura física, la ambiental, la administrativa, la fiscal, el desarrollo del capital humano y social.

- Participación comunitaria. Parte del reconocimiento de la capacidad que tienen 
las organizaciones sociales de las comunidades afectadas para asumir su propia recuperación, con la ayuda de las organizaciones estatales y privadas orientadas al servicio social, en donde cada uno de los intervinientes se considera como un actor igualmente relevante.

- Transparencia. Es una apuesta por el manejo claro de los recursos públicos, como una manera de luchar contra la corrupción y de evitar la injerencia politiquera en el manejo de estos.

- Celeridad. Se refiere a la necesidad de ajustar los plazos a los previstos o disminuirlos, de acuerdo con las disponibilidades de los recursos, siempre y cuando se obtengan los resultados buscados.

- Eficiencia. Encaminada a la utilización óptima de los recursos, es decir, al uso adecuado de los mismos en términos de los niveles y racionalidad del gasto, cumplimiento de los plazos exigidos y de la calidad de las actividades y de sus resultados.

- Sostenibilidad. Este principio propende a un pensamiento que trascienda el aquí y el ahora, para llevar a la reflexión del mediano y largo plazo. Toda acción debe ser vista en la dimensión del desarrollo $y$, por lo tanto, requiere pensar en involucrar orgánicamente los elementos del sistema que se encuentren débilmente vinculados o que, en un momento dado, se rezaguen en su función, en aras de garantizar la permanencia del desarrollo.

- Descentralización. Si bien es cierto que la política de la reconstrucción hace referencia a objetivos y aspectos puntuales, estos se consideran como criterios y orientaciones que facilitan dar cuenta de las particularidades de las localidades y de las comunidades. Igualmente, propicia la interacción con las autoridades de las entidades territoriales, en el ánimo de contribuir con los principios constitucionales de respeto a la autonomía territorial, la subsidiariedad y la complementariedad de los recursos públicos.

El modelo vincula entre sí a los diferentes componentes de la sociedad civil organizada de cada municipio, y les confiere facultades para actuar en nombre del Forec, bajo las orientaciones de los gobiernos departamental y municipal, en el desarrollo de las operaciones de planeación, diseño y ejecución de los proyectos dirigidos a atender la recuperación de la infraestructura física, la generación de empleo, la reactivación económica, la atención humanitaria y social y la reconstrucción del tejido social.

\section{Ejes de la reconstrucción y líneas estratégicas de intervención}

De la misma manera como se elaboró el punto anterior, los ejes propuestos desde la política y la normatividad se interpretan a la luz de los avances del proceso. Ellos son:

- Vivienda y restitución de equipamientos públicos. En su primera parte, este eje tiende a restablecer los derechos individuales perdidos o vulnerados por el sismo, es decir, la vivienda; en su segunda parte, en tanto, procura recuperar la locación institucional que permita normalizar la prestación de los servicios básicos y regulares de apoyo a la vida de los ciudadanos en una localidad o municipio.

- Fortalecimiento del tejido social. Se entiende como el fortalecimiento de la sociedad de las distintas localidades, 
tendiente a insertar a las comunidades en procesos organizativos consolidados y con transferencia de conocimientos.

- Reactivación económica. Puede ser entendida desde tres puntos de vista. El primero se refiere a los beneficios directos para la región en términos de generación de ingresos, empleo, empresas y negocios, que resultan de la inversión de cerca de $\$ 1,6$ billones en los distintos aspectos de la reconstrucción. El segundo hace alusión a los elementos contemplados en los Decretos 258 y 350 de 1999, que consideran el estímulo al sistema económico y la promoción de empresas en los municipios, a partir del otorgamiento de beneficios en materia de impuestos, tasas, gravámenes, derechos y contribuciones parafiscales. El tercer punto de vista tiene que ver con la vía de la aplicación de los recursos previstos en proyectos específicos de carácter productivo.

- Recuperación ambiental. Pretende rescatar la necesidad de (re)pensar el Eje Cafetero como una región de alta vulnerabilidad sísmica y ambiental y, por lo tanto, plantea que cualquier acción que se emprenda debe consultar instrumentos básicos de ordenamiento territorial que disminuyan el riesgo físico y la vulnerabilidad social.

- Politicas públicas. Este eje igualmente puede ser abordado por lo menos de dos maneras. De un lado, en función del apoyo mutuo y la coordinación con las entidades descentralizadas de orden territorial, departamentos y municipios, en cabeza de las cuales se sustenta la institucionalidad permanente del Estado. En la fase conclusiva de la recons- trucción por parte del Forec, explícitamente se proponen medidas tendientes a la transición y transferencia del proceso reconstructivo desde el Fondo y las Gerencias Zonales hacia las entidades territoriales, para lo cual se empeña en contribuir a su fortalecimiento institucional e incremento de capacidad, de manera que puedan asumir estas nuevas responsabilidades. De otro lado y en la misma perspectiva de las políticas públicas, entendidas estas como la manera de decidir sobre necesidades colectivas, el Fondo se propuso desde el principio promover y ampliar la participación de la sociedad civil y apoyar las maneras de organización y participación autónomas de las comunidades que a través de estos cuerpos se posicionaban y reclamaban interlocución con el Forec.

\section{Vinculación de las ONG y los contra- tos de administración delegada}

Desde el mismo momento del desastre y en las fases más críticas de la emergencia, las ONG nacionales e internacionales hicieron presencia en las áreas de mayor interés público: unas se emplearon a fondo en la búsqueda de supervivientes y en atención a los heridos; otras se ocuparon del saneamiento ambiental; muchas estuvieron atentas a la alimentación básica de los damnificados. No pocas cumplieron con múltiples tareas en los campos del medio ambiente (Gómez, 2001b).

Las organizaciones sociales que acudieron a la zona, inspiradas en un enfoque "emergente” de la gestión pública, acordaron con el Estado una organización para acometer la reconstrucción, la cual fue aprobada por el Consejo Directivo del Fondo el 17 de febrero de 1999, de acuerdo con el Acta n. ${ }^{\circ} 2$. Se vincularon diferentes tipos de organizacio- 
nes: gubernamentales, no gubernamentales comunitarias, estatales, municipales, gremios de la construcción, empresas industriales y comerciales del Estado, firmas de ingeniería, organizaciones internacionales como el Banco Mundial, Banco Interamericano de Desarrollo (BID), Ayuda Internacional para el Desarrollo (AID), Programa de las Naciones Unidas para el Desarrollo (PNUD), embajadas, organizaciones comunitarias, fundaciones sin ánimo de lucro, etcétera.

Bajo la denominación genérica de ONG, en las Gerencias se agruparon múltiples realidades. Se tuvo la presencia de Organizaciones de Vivienda Popular de larga tradición de trabajo comunitario y ciudadano (Fenavip o Fedevivienda), pasando por organizaciones que actúan bajo la tutela empresarial (Antioquia Presente o Carvajal), hasta consorcios que se crearon específicamente para operar en condiciones de Gerencias Zonales: los ocho municipios del Norte del Valle se atendieron con un Plan Zonal gerenciado por el Fondo para la Solidaridad del Valle, una organización que representa un acuerdo entre varias ONG. En Pereira, la Fundación Vida y Futuro fue un acuerdo entre la Cámara de Comercio y Confamiliares. Las Universidades Nacional y de Antioquia operaron como gerentes zonales en Armenia. La Federación Nacional de Cafeteros tuvo los contratos para reconstruir la zona rural cafetera y no cafetera. La Sociedad Colombiana de Arquitectos respondió por la reconstrucción de escuelas y colegios.

\section{El proceso de reconstrucción por fases}

Para efectos de atender a la población y a los municipios afectados, se definieron tres fases de atención:

- Emergencia, para atender transitoria- mente las necesidades vitales; incluyó la fase de Transición y Planificación, y la Fase de Recuperación y Desarrollo de la zona. Igualmente, y de acuerdo con el nivel de daño y la magnitud de la tragedia, el gobierno nacional estableció un orden de prioridades para orientar la asignación de los recursos; el orden fue: vivienda y edificaciones públicas, equipamiento urbano, infraestructura de transporte; educación, cultura y recreación; salud, servicios públicos domiciliarios, fuerzas militares y de policía; medio ambiente; tejido social; desarrollo productivo, reactivación económica, generación de empleo; desarrollo rural, ordenamiento territorial; planificación y prevención de desastres, gestión del riesgo y gestión institucional.

- Planeación, la segunda etapa es de consolidación, y corresponde al periodo de diagnóstico de los daños ocurridos en la zona y a la planificación para la reconstrucción. Este proceso se expresó en la elaboración de los Planes de Acción Zonal, instrumentos que contenían los proyectos y la estrategia para su ejecución.

- Reconstrucción, la tercera etapa, es la correspondiente a la implementación del proceso de contratación con transparencia y eficiencia, para lo cual se aplicó la contratación entre privados y bajo el reglamento operativo del Banco Mundial y del BID. En esta etapa se ejecutaron los proyectos.

Finalmente, se diseñó una estrategia que permitió la liquidación y supresión del Forec, como resultado de la finalización de las actividades y funciones encargadas. Esta 
última fase se desarrolló bajo los lineamientos expresados por el Consejo Nacional de Política Económica y Social (Conpes) en el Documento 3131 de septiembre de 2001, y los mismos principios con que actuó el Forec durante su ejecución, en especial los de eficiencia, celeridad y transparencia.

\section{Palabras finales: el inicio de una tarea política y cultural}

Los ciudadanos son los que hacen las ciudades, y aunque las personas se encuentran con territorios y hábitats que las condicionan desde que nacen, para bien o para mal, estos espacios acaban siendo transformados por los humanos. La sustentabilidad incluye cuanto vaya a pasar en futuras generaciones y, por lo mismo, se basa en la cultura cívica de eso que hacemos hoy y de aquello que harán mañana nuestros descendientes. En todo caso, parece que solo es posible hablar de experiencias en los hábitats cuando hacemos y construimos ciudad con ciudadanía.

En este orden de ideas, la dimensión política y la participación tienen que servir para la integración social, para proyectos sustentables y adaptados a las características concretas de cada territorio. Nos referimos a una participación que genere inclusión social, integrada a una creciente expansión de la ciudadanía, donde actuemos como seres sociales y no individuales.

Cuando se asume como proyecto existencial una actitud que pone en cuestión cualquier ámbito del statu quo, es importante tener en cuenta que, de un lado, invariablemente ella generará - nos demos cuenta o no- algún tipo de rechazo en el ámbito del establecimiento contra el cual se dirige la intención de cambio; y del otro, que también — seamos conscientes o no de ello- esa ac- titud jamás quedará impune: la institución establecida tarde o temprano pasará cuenta, no solo por aquello que se hace en contra de la dominación vigente, sino incluso por el atrevimiento de permitirse pensar que se podría, o se debería cambiar. Por lo que en biología se denomina autopoiesis, la institución atacada no puede dejar prosperar nunca, especialmente en su interior, ningún proceso que pueda impedir la garantía de su propia reproducción.

El terremoto mostró toda la precariedad de nuestra sociedad para reaccionar y atender la emergencia, y también dejó al desnudo la pobreza intelectual y material sobre la cual está construida nuestra realidad urbana y rural, pero en ningún momento ello significó que la capacidad de defender las formas de dominación sobre las cuales se ha mantenido hayan sufrido la más mínima fisura.

El sismo derrumbó la edificación arquitectónica y urbanística y arrasó con la infraestructura; tumbó la ciudad, pero, aunque profundizó los niveles de pobreza de los pobres y los excluidos, no tocó el poder de sometimiento (el cual, también, está entronizado en la mente, en la psiquis de los hombres y mujeres) que mantiene las bases de cuanto es hoy la realidad socio-histórica conocida como el Eje Cafetero, ni mucho menos la de Colombia.

Ante esa situación de destrucción y de tragedia, que pedía desesperadamente referentes para reedificar la existencia, para refundar la ciudad, hicieron bien en jugársela con sus propuestas (en estricto sentido, tampoco tenían otra posibilidad: era inevitable la apuesta) esos hombres y mujeres de la región que, desde mucho antes del terremoto, pensaban que era bello construir su opción de vida alrededor de configurar una 
propuesta de urbe distinta a aquella que se derrumbó hace un poco más de cuarenta meses; e hicieron muy bien en buscar, como buscaron, todos los ámbitos y momentos para tratar de colocar su proyecto y para que fuese aceptado como modelo.

Apostar por una ciudad justa, equitativa, creativa, sostenible, democrática y potente económica y culturalmente en Colombia, no es un ejercicio académico o profesional cuyo tránsito pueda pasar indemne en un medio como el nuestro, donde la urbe que tenemos es el resultado y la condensación de unas formas de dominación sustentadas en la violencia, la exclusión y la irresponsabilidad de las relaciones con la naturaleza.

Y lo que quedó después de la tragedia no fue un campo arrasado, en el cual se podía venir a construir otro proyecto soportado "únicamente" por la lógica de demostrar racionalmente que era más democrático, más eficiente, más sostenible y más bello. Fue un terreno abierto donde las fuerzas tradicionales (de todo tipo) de dominación y el conformismo generalizado, también quieren (volver a) utilizar su poder político, económico e ideológico para reeditar la construcción de un aglomerado de materiales que ya demostró que era absolutamente rentable para (y acomodado a) sus intereses inmediatos y particulares.

En definitiva, la consideración según la cual la política tiene un ámbito autónomo y se ocupa solo del control y dominio del poder público, evade el hecho de que la participación de las comunidades, además de tener un carácter histórico, tiene una función transformadora de la sociedad, y por ello es en esencia un ejercicio pleno de la política. Es necesario, por tanto, abordar el tema de lo político y del ejercicio de la política en su expresión más auténtica, tal como la plantea Castoriadis (1996): "Ahora bien, la política no existe siempre y en cualquier lugar; la verdadera política es la resultante de una creación histórico-social rara y frágil. Lo que existe en toda sociedad es lo político: la dimensión -explícita, implícita o quizá casi imperceptible- que tiene que ver con el poder; esto es, la instancia o instancias instituidas que pueden emitir mandatos con autoridad y que, al menos, deben incluir siempre, de forma explícita, lo que denominamos un poder judicial y un poder de gobierno".

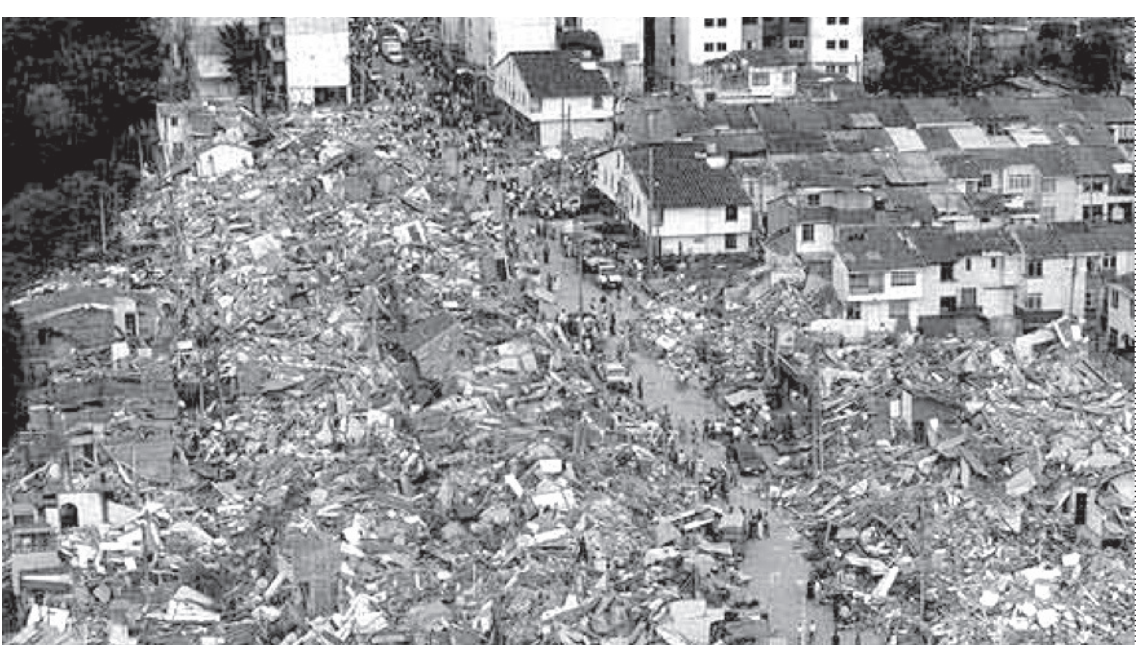




\section{Referencias bibliográficas}

Arango, O. (2006). Refundar el pacto por la región. Capacidad institucional en el Eje Cafetero. Perspectivas para un segundo Informe de Desarrollo Humano en el Eje Cafetero. Pereira, Colombia: Programa de las Naciones Unidas para el Desarrollo (PNUD).

Castoriadis, C. (1996). La democracia como procedimiento y como régimen. Iniciativa Socialista (Madrid), n. 38 .

Gómez, D. (2001a). Análisis preliminar del Modelo de Gestión de la Reconstrucción en el Eje Cafetero. Armenia, Colombia: Fondo para la Reconstrucción Social y Económica del Eje Cafetero (Forec) / Centro Interdisciplinario de Estudios sobre Desarrollo (Cider), Universidad de los Andes, Colombia. Documento preparado para ser presentado en el Encuentro Nacional La Región en la Colombia del siglo XXI, Bogotá, octubre de 2001.

Gómez, D. (2001b). Hitos del proceso de la reconstrucción. Documento de trabajo para el Monitoreo Social de la Red de Universidades, mayo de 2001.

Echeverri Castrillón, L. \& J. Rodríguez Rodríguez (2002). Planificación, vivienda, desarrollo urbano y medio Ambiente. Una lectura del proceso de reconstrucción del eje cafetero colombiano. Armenia, Colombia: Fondo para la Reconstrucción Social y Económica del Eje Cafetero (Forec) / Centro Interdisciplinario de Estudios sobre Desarrollo (Cider), Universidad de los Andes, Colombia / Banco Mundial.

Habermas, J. (1998). La inclusión del otro. Estudios de teoría política. Barcelona: Editorial Paidós.

Martín-Barbero, J. (2000) Comunicación y cultura politica. Conferencia ofrecida en Armenia en el marco de las Tertulias preparadas por Viva la Ciudadanía. Mimeógrafo. Armenia, Colombia.

Rorty, R. (1989). Contingencia, ironia y solidaridad. Barcelona: Editorial Paidós.

Rodríguez, G. (2005). Ciudad-región del Eje Cafetero. Hacia un desarrollo urbano sostenible. Pereira, Colombia: Gráficas JES.

Rodríguez J. (2000). La vivienda en busca de un hermoso y rebelde sueño. Bogotá: Editorial Universitaria de Colombia.

Rodríguez, J. (2004) El presupuesto participativo en Risaralda. Defendiendo lo público. Experiencia metodológica y resultados. Pereira, Colombia: GTZ / Universidad de Málaga, España.

Rodríguez J. (2007) El presupuesto participativo en Manizales: Un aprendizaje en la construcción de la ciudadanía. Ánfora (Universidad Autónoma de Manizales), n. 19.

Sánchez. J. (2000). Participación ciudadana: un derecho para el nuevo milenio. Revista del Taller de la Ciudad, CIVITAS (Armenia, Colombia), n. ${ }^{\circ}$. 